\title{
Concurrent granulomatous Pneumocystis carinii and Mycobacterium xenopi pneumonia: an unusual manifestation of HIV immune reconstitution disease
}

\author{
F Chen, G Sethi, R Goldin, A R Wright, C J Lacey
}

Thorax 2004;59:997-999. doi: 10.1136/thx.2003.012567

This report of concurrent granulomatous Pneumocystis carinii pneumonia (GPCP) and Mycobacterium xenopi pneumonia (MXIP) in a patient with advanced HIV disease $3-5$ weeks after commencing antiretroviral therapy (ART) fits the syndrome of HIV immune reconstitution/restoration disease (IRD). It may also be a unique window into the immunology of granulomatous inflammation.

\section{A} 35 year old white HIV positive man commenced antiretroviral therapy (ART) with zidovudine, lamivudine, and nelfinavir 7 years after diagnosis (CD4 count 20 cells/ml, viral load 154000 copies/ml (Chiron III)). Prophylaxis for Pneumocystis carinii pneumonia (PCP) was with nebulised pentamidine. Three weeks after commencing ART, with no prior respiratory symptoms, he developed an unproductive cough. Despite 2 weeks of treatment with amoxicillin, his cough persisted and, although he was well, a chest radiograph 5 weeks after initiating ART showed bilateral apical consolidation (fig 1A). Arterial blood gas tensions on air were normal $\left(\mathrm{PCO}_{2} 4.53 \mathrm{kPa}, \mathrm{PO}_{2} 16.3 \mathrm{kPa}\right.$, $\mathrm{pH}$ 7.468). Bronchoscopic examination after 3 days of treatment with high dose intravenous Septrin showed generalised mucosal inflammation, and the bronchoalveolar lavage (BAL) fluid had scanty Candida albicans but was negative for acid fast bacilli and $P$ carinii. Ten days after PCP treatment the apical consolidation improved significantly (fig 1B) but the appearance of a new area of right mid-zone consolidation led to diagnostic biopsy to exclude a malignant process. A percutaneous computed tomographic (CT) guided lung biopsy (left apex, $3 \times 20 \mathrm{G}$ tissue cores) was undertaken 13 days after admission. The CT scan showed consolidation (fig IC and D) with multiple small and medium sized pulmonary nodules (many cavitated) in both upper and right middle lobes. Histological examination showed granulomatous PCP (fig IE and F). Blood culture for $M$ avium complex and polymerase chain reaction for $M$ tuberculosis were both negative. $M$ xenopi was isolated from the BAL fluid and three sputum specimens after 6 weeks but tissue culture was negative. Precautionary empirical treatment for $M$ tuberculosis with rifampicin, isoniazid, pyrazinamide and ethambutol was commenced after the biopsy finding of granulomatous lung disease but was discontinued after 6 weeks, and nontuberculous mycobacterial treatment with clarithromycin, ciprofloxacin and rifabutin was started and continued until resolution of the right middle lobe abnormalities 14 months later. The patient remains well on ART with a CD4 count of 510 cells/ml and an undetectable viral load.

\section{DISCUSSION}

Race et al $^{1}$ reported five patients who, within weeks of commencing ART, developed a systemic syndrome with marked inflammatory reactions to latent infection with $M$ avium complex. All experienced CD4 increments. Such paradoxical hyper-accentuated inflammatory responses to pre-existing or co-existing infections are recognised complications of immune reconstitution following ART; occurring within days to months and invariably associated with CD4 increments, they are collectively known as immune reconstitution/restoration disease (IRD). ${ }^{2}$ The dynamics of $\mathrm{T}$ cell increments following ART is a biphasic phenomenon; ${ }^{3}$ the first phase (weeks to 6 months) is predominantly of $\mathrm{T}$ memory cell redistribution, while the second phase (usually $>6$ months) is mainly of naïve CD4 cell reconstitution. As IRD invariably coincides with the primary phase of T memory cell redistribution, it has been postulated that this may be a memory $\mathrm{T}$ cell versus pathogen/antigen recognition event.

Mycobacterium xenopi infections have been, uncommonly, reported as IRD. ${ }^{4}$ Because co-existence of multiple opportunistic organisms in the respiratory tract of patients with advanced HIV disease is common, ${ }^{5}$ diagnosing non- $M$ tuberculosis infections can be difficult; in our patient the isolation of $M$ xenopi from BAL fluid and sputum specimens and the clinical features all fit the diagnostic criteria for non$M$ tuberculosis infections. ${ }^{6}$ The differential time scales for the resolution of the apical radiographic abnormalities (rapid diminution after 10 days of PCP therapy) and the radiographic abnormalities of the right middle lobe ( 14 months) are compatible with concurrent granulomatous $P$ carinii pneumonia (GPCP) and $M$ xenopi pneumonia (MXIP).

The granuloma, a localised inflammatory process, can form part of the host's immune protection response to infections or part of an autoimmune disease process. Patients with AIDS have $\mathrm{T}$ cell deficiency and consequently have difficulty in mounting a granulomatous reaction to infections. ${ }^{7}$ Despite this, our patient mounted a hyper-accentuated response weeks after commencing HAART.

Bondoc et $a l^{8}$ reported GPCP in three non-HIV patients with malignancy who shared similarities with our patient. GPCP occurred 2 weeks to 2 months after stopping corticosteroids compared with 3-8 weeks after receiving ART in our patient. All had minimal symptoms, a comparable time scale of immune reconstitution, and $P$ carinii was not detected in the BAL fluid. These two reports seem to support recent evidence that the main pathway for $\mathrm{T}$ cell recruitment during granuloma formation is from the systemically activated $\mathrm{T}$ cell pool. ${ }^{9}$ Corticosteroid therapy suppresses peripheral circulating lymphocytes and its discontinuation leads to a rebound of peripheral lymphocytes, ${ }^{10}$ a process akin to the phase of memory $\mathrm{T}$ cell redistribution following ART.

The simultaneous occurrences of GPCP and MXIP are perhaps less surprising when viewed in the context of $\mathrm{T}$ memory cell (therefore diverse memory clone) redistribution. Recent evidence has shown that the $\mathrm{T}$ cell repertoire of granulomas in response to a primary pathogen is diverse. 

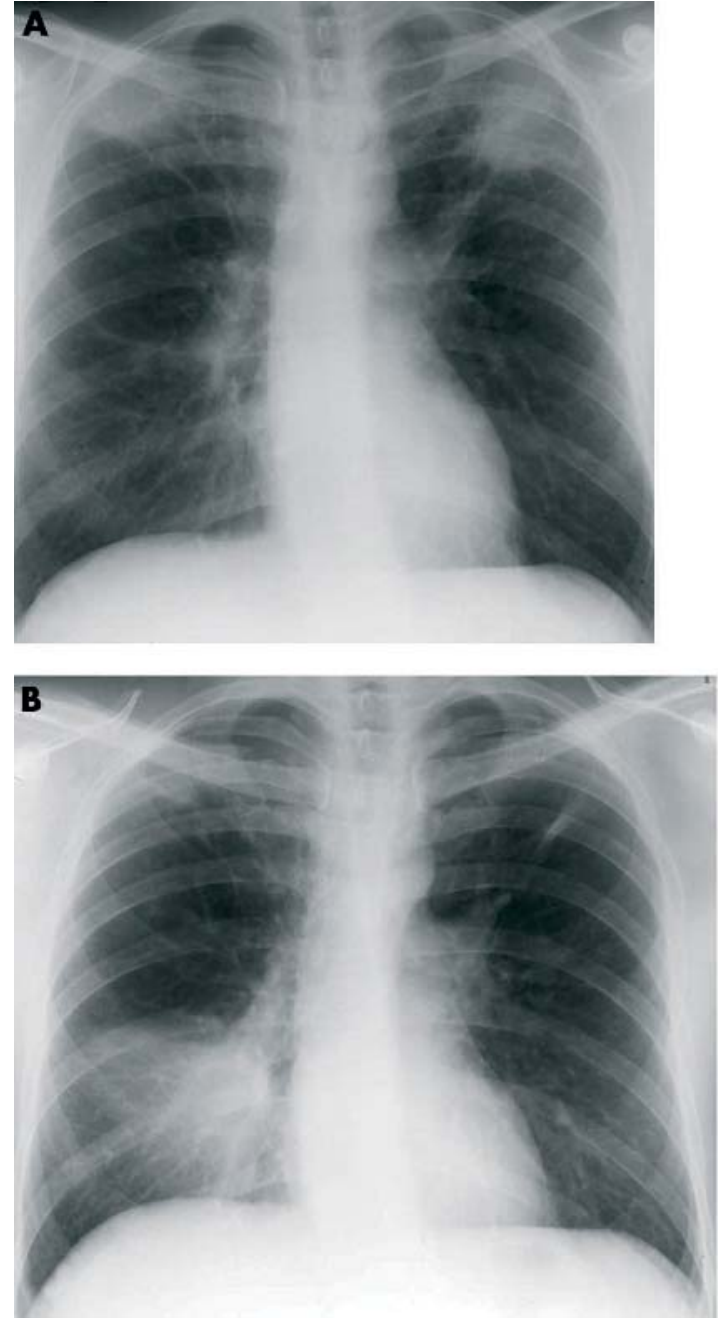
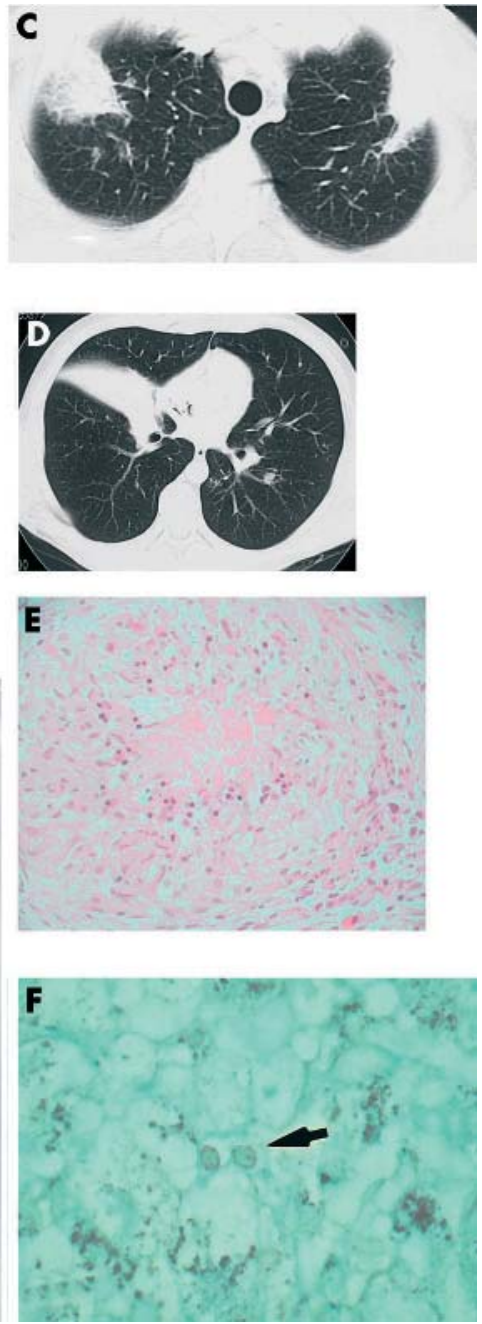

Figure 1 Chest radiographs taken (A) 5 weeks after starting ART showing bilateral apical consolidation and (B) 7 weeks after starting ART showing diminished apical consolidation following 10 days of treatment with Septrin but with a new area of right middle lobe consolidation. $M$ xenopi was subsequently isolated from BAL specimens and from three sputum cultures. (C), (D) CT scans of the thorax during percutaneous lung biopsy 8 weeks after starting ART which delineate the chest radiographic abnormalities and also show small and medium sized pulmonary nodules. (E) Nonnecrotising pulmonary granuloma from a representative sample of a percutaneous CT guided biopsy specimen of the left lung apex (20G cores $\times 3$; H\&E stain; magnification $\times 10$ ). (F) Owl's eyes appearance of Pneumocystis carinii (arrowed) identified from a representative sample biopsy of the left lung apex (Grocott stain; magnification $\times 40$ ).

Only $30-60 \%$ in a single granuloma are specific to the inducing antigen, and systemically activated T cells with no specificity to the granuloma also have access to it. ${ }^{9}$

Cytokine gene polymorphisms ${ }^{11}$ and an abnormal cytokine profile ${ }^{12}$ have been associated with HIV IRD. This, together with a recent report of granulomatous pulmonary disease occurring in a patient following cessation of tumour necrosis factor (TNF)- $\alpha$ antagonist treatment, ${ }^{13}$ implies that perturbation of the cytokine profile can trigger granulomatous inflammation.

Host and antigenic factors implicated in the pathogenesis of granulomatous lung diseases are also likely to influence the expression of GPCP; in addition, immune dysregulation (systemic $\mathrm{T}$ cell pool and cytokine perturbation) may also trigger granulomatous inflammation.

\section{ACKNOWLEDGEMENTS}

The authors thank Drs G Brook and M Murphy for their critical comments and suggestions.

\section{Authors' affiliations}

F Chen, Department of Sexual Health, Whipps Goss University Hospital and Department of Infection and Immunity, St Bartholomew's Hospital,
London, UK (formerly Department of Genitourinary Medicine, St Mary's Hospital, London, UK)

G Sethi, Department of Genitourinary Medicine, St Mary's Hospital, London, UK

R Goldin, Department of Histopathology, St Mary's Hospital, London, UK

A R Wright, Department of Radiology, St Mary's Hospital, London, UK C J Lacey, Reader in Infectious Diseases, Hull York Medical School, University of York, UK (formerly Imperial College London and

Department of Genitourinary Medicine, St Mary's Hospital, London, UK)

Correspondence to: Dr F Chen, C/O Andrewes Unit, St Bartholomew's Hospital, London EClA 7BE, UK; fchenff@msn.com

Received 29 July 2003

Accepted 10 December 2003

\section{REFERENCES}

1 Race EM, Adelson-Mitty J, Kriegel GR, et al. Focal mycobacterial lymphadenitis following initiation of protease-inhibitor therapy in patients with advanced HIV-1 disease. Lancet 1998;351:252-5.

2 DeSimone JA, Pomerantz RJ, Babinchak TJ. Inflammatory reactions in HIV-1infected persons after initiation of highly active antiretroviral therapy. Ann Intern Med 2000;133:447-54. 
3 Autran B, Carcelain G Li TS, et al. Positive effects of combined antiretroviral therapy on CD4+ T cell homeostasis and function in advanced HIV disease. Science 1997;277:112-6.

4 Bachmeyer C, Blum L, Stelianides S, et al. Mycobacterium xenopi pulmonary infection in an HIV infected patient under highly active antiretroviral treatment. Sex Transm Infect 2002;78:60-1.

5 el Helou P, Rachlis A, Fong I, et al. Mycobacterium xenopi infection in patients with human immunodeficiency virus infection. Clin Infect Dis 1997:25:206-10.

6 Ahn CH, McLarty JW, Ahn SS, et al. Diagnostic criteria for pulmonary disease caused by Mycobacterium kansasii and Mycobacterium intracellulare. Am Rev Respir Dis 1982;125:388-91.

7 Perlman DC, El Helou P, Salomon N. Tuberculosis in patients with human immunodeficiency virus infection. Semin Respir Infect $1999 ; 14: 344-52$.
8 Bondoc AY White DA. Granulomatous Pneumocystis carinii pneumonia in patients with malignancy. Thorax 2002;57:435-7.

9 Hogan $L H$, Wang $M$, Suresh $M$, et al. CD4+ TCR repertoire heterogeneity in Schistosoma mansoni-induced granulomas. J Immunol 2002;169:6386-93.

10 Tornatore KM, Venuto RC, Logue G, et al. CD4+ and CD8+ lymphocyte and cortisol response patterns in elderly and young males after methylprednisolone exposure. J Med 1998;29:159-83.

11 Price $P$, Morahan G, Huang D, et al. Polymorphisms in cytokine genes define subpopulations of HIV-1 patients who experienced immune restoration diseases. AIDS 2002;16:2043-7.

12 Stone SF, Price P, Keane NM, et al. Levels of IL- 6 and soluble IL-6 receptor are increased in HIV patients with a history of immune restoration disease after HAART. HIV Med 2002;3:21-7.

13 Vavricka SR, Wettstein T, Speich R, et al. Pulmonary granulomas after tumour necrosis factor alpha antagonist therapy. Thorax 2003;58:278-9.

\section{LUNG ALERT}

\section{Sildenafil and exercise capacity in hypoxic pulmonary hypertension}

$\Delta$ Ghofrani HA, Reichenberger F, Kohstall MG, et al. Sildenafil increased exercise capacity during hypoxia at low altitudes and at Mount Everest base camp. Ann Intern Med 2004;141:169-77

T

his study examined the influence of oral sildenafil on pulmonary haemodynamics and exercise tolerance during hypoxia induced pulmonary hypertension in healthy mountaineers and trekkers who were not susceptible to high altitude pulmonary oedema. Fourteen healthy volunteers (12 men, median age 36.5 years) were enrolled and randomised in a double blind, placebo controlled, crossover design. Participants were assigned to receive placebo or one $50 \mathrm{mg}$ dose of sildenafil. Systolic pulmonary artery pressure, cardiac output, and peripheral arterial oxygen saturation at rest and during assessment of maximum exercise capacity on cycle ergometry were measured, both while breathing a hypoxic gas mixture with $10 \%$ fraction of inspired oxygen at low altitude and then at the Mount Everest base camp (5400 m).

Sildenafil reduced hypoxic pulmonary hypertension at rest and with exercise, and increased maximum exercise capacity and cardiac output. The study did not examine the effects of sildenafil on normoxic exercise tolerance, and the authors cautioned about the unclear role of sildenafil in the management of acute mountain sickness. In addition, it is not known whether sildenafil increases exercise capacity in hypoxic pulmonary hypertension due to lung diseases such as COPD.

M M Mughal

The Cleveland Clinic Foundation, Cleveland, Ohio, USA; mughalm@ccf.org 